\title{
Characteristics of HIV Antiretroviral Regimen and Treatment Adherence
}

Vera Lúcia da Silveira, Maria de Lourdes Drachler, José Carlos de Carvalho Leite and

Cézar Arthur Tavares Pinheiro
Faculty of Medicine of the Federal University of Pelotas and Municipal Secretary of Health of Pelotas; University of Caxias do Sul, Rio Grande do Sul, Brazil

The relationship between characteristics of HIV antiretroviral regimens and treatment adherence was studied in adolescent and adult patients who underwent antiretroviral therapy from January 1998 to September 2000, at the Service for Specialized Assistance in Pelotas. The patients were interviewed on two occasions, and the use of antiretrovirals during the previous 48 hours was investigated by a self-report. Adherence was defined as use of $95 \%$ or more of the prescribed medication. Social-demographic variables were collected through direct questionnaires. The antiretroviral regimen and clinical data were copied from the patients' records. Associations between the independent variables and adherence were analyzed by means of logistic regression. The multivariate analysis included characteristics of the antiretroviral regimens, socialdemographic variables, as well as perception of negative effects, negative physiological states, and adverse effects of the treatment. Among the 224 selected patients, 194 participated in our study. Their ages varied from 17 to 67 years; most patients were men, with few years of schooling and a low family income. Only $49 \%$ adhered to the treatment. Adherence to treatment regimens was reduced when more daily doses were indicated: three to four doses (odds ratio of adherence to treatment $(\mathrm{OR})=0.47,95 \%$ confidence interval $(\mathrm{CI}) \mathbf{0 . 2 2}-1.01)$ and five to $\operatorname{six}(\mathrm{OR}=\mathbf{0 . 2 4 , 9 5 \%} \mathrm{CI}$ 0.09-0.62); two or more doses taken in a fasting state $(\mathrm{OR}=0.59,95 \% \mathrm{CI} 0.11-0.68)$, and for patients who reported adverse effects to the treatment $(\mathrm{OR}=0.39,95 \% \mathrm{CI} 0.19-0.77)$. Most of the regimens with more than two daily doses of medication included at least one dose apart from mealtimes. The results suggest that, if possible, regimens with a reduced number of doses should be chosen, with no compulsory fasting, and with few adverse effects. Strategies to minimize these effects should be discussed with the patients.

Key Words: HIV/AIDS, adherence, antiretroviral therapy.

HIV infection is a serious public health problem. According to estimates from the World Health Organization (WHO), in 2000 there were more than

Received on 11 September 2002; revised 04 January 2003. Address for correspondence: Dr. Vera Lúcia da Silveira. Rua Barão de Azevedo Machado, 105, apto 402 A, Pelotas-RS, Zip code: 96020150, Brazil. E-mail: vlsil@zaz.com.br. Phone numbers: 0XX53-2788034- 0XX53-2278236-0XX539816756. Sources of financing: Fundação de Amparo à Pesquisa do Estado do Rio Grande do Sul (Research Support Foundation of the State of Rio Grande do Sul). Conselho Nacional de Pesquisa (CNPq) (National Council of Scientific and Technological Development).

The Brazilian Journal of Infectious Diseases 2003;7(3):194-201 (C) 2003 by The Brazilian Journal of Infectious Diseases and Contexto Publishing. All rights reserved.
36 million infected people and 22 million had already died [1]. Another 5.3 million were infected in 2000, which means more than 15,000 people per day or 11 per minute [2].

Antiretroviral therapy has changed the prognosis of patients infected with HIV [3]. This treatment causes a reduction of viral load count to undetectable levels [3], thus reducing the incidence of infections [4,5], hospitalizations [5], and mortality [4,5]. Treatment effectiveness requires high levels of adherence [5-8]. RNA levels higher than 400 copies/ml were found in only $22 \%$ of the patients whose treatment adherence was $\geq 95 \%$; on the other hand these high levels were found in $80 \%$ of the patients whose adherence was $<80 \%$ [5]. Other studies have also found low adherence 
to be associated with detectable viral load (> 400 [9] or $500 \mathrm{copies} / \mathrm{ml}$ [7]), and with cross-resistance to other antiretrovirals $[6,8,10]$. Cross-resistance interferes with future therapeutic options for the individual being treated and for those who subsequently become infected with the resistant virus. Therefore, low adherence can have a devastating effect on the individual and can also pose an even greater problem for public health.

Nowadays, according to the Brazilian Ministry of Health, an adherent patient is defined as one who takes $\geq 95 \%$ of the prescribed dose [1,5,7]. However, a significant proportion of patients do not reach high levels of adherence. In one study, $37 \%$ of the patients used less than $80 \%$ of the prescribed medication [11]. More recent data reveal that $31 \%$ of the patients took $\leq 90 \%$ of the doses [12]. In Brazil, it is estimated that $30-40 \%$ of all patients do not adhere to treatment (they take less than $80 \%$ of the medication prescribed). These data were obtained by means of a self-report analysis [13].

The effect of the characteristics of the antiretroviral regimen and treatment adherence has been studied [12, 14-22]. Increased numbers of antiretroviral drugs used in the treatment regimens is expected to make it more difficult for the patient to follow the prescription. A majority of trials confirm this hypothesis [16,18-21, 23-25]. However, there was no evidence of such an effect in other studies [7,11-13,22,26-28].

The number of doses is another important variable. Some studies support the hypothesis that the number of doses is more important than the number of drugs used $[16,18,28]$, as a small number of medication sessions tends to fit more easily into the patient's daily routine [19,24,28-30]. Nevertheless, other studies did not find this association [12,13,22], which suggests a need for more comprehensive studies concerning this issue.

The effect of the number of pills has also been studied. Taking a larger number of pills is likely to affect adherence due to the greater difficulty involved $[18,21,24,28]$. In one study [19], the difficulty of taking a large number of pills was the fourth most frequent cause of non-adherence; it was reported by $50 \%$ of the non-adherent patients. Other authors did not find a link between this variable and adherence [4,11$13,15,30,31]$; thus, the possibility of an effect of the number of pills on adherence was deemed inconclusive.

Some antiretroviral regimens require that the medication be taken with food, during a hearty meal or in a fasting state $[19,24,32-34]$ in order to attain full absorption. In one study [19], the primary cause of non-adherence was hunger, due to the fasting requirement. In another study [25], 23\% of the patients reported difficulty in following this recommendation. Although no studies were found involving taking medication with meals, adherence is expected to be higher in this case.

Most of the review studies [18,34,35] and research papers $[4,19,36-38]$ on this subject suggest that adverse effects are contributing factors to nonadherence. Other factors, such as little schooling [13,21], emotional dysfunction (anxiety [21] and depression [11,39-41], were also considered risk factors for non-adherence.

We examined the effect of antiretroviral regimen characteristics and treatment adherence in an HIV/ AIDS reference center in southern Brazil.

\section{Materials and Methods}

Design and data collection procedures. We studied all patients aged 13 and over registered at the Service of Specialized Assistance for HIV/AIDS of the city of Pelotas-RS (SAE), who were prescribed antiretroviral therapy for treatment of HIV infection from January 1998 to September 2000.

Data collection took place between May and December of 2000; it included two interviews with each patient. Well-trained personnel interviewed the patients after obtaining written consent. Interviews took place at the SAE, when patients went there for a medical appointment or to obtain their medication at the center's pharmacy. Patients who did not attend the outpatient clinic $(n=53)$ were interviewed at home and were advised to return to the outpatient clinic.

During the first interview, patients answered a questionnaire that included social-demographic data, 
the use of antiretrovirals and other medications, as well as perception of adverse effects to antiretroviral treatment, of negative effects and of negative physiological states. The second interview, with a minimum interval of one month after the first one, investigated the use of antiretrovirals and other medications. Two medical researchers, experts in HIV/ AIDS, copied all data on clinical evaluations, duration of treatment and use of antiretrovirals directly from the patients' records. The variables studied were:

Social-demographic characteristics. Data that included social details, such as age, gender, formal education and family income were collected with semiopen questions.

\section{Clinical, viral and immunological characteristics.} Current viral load was estimated by means of the serum HIV-RNA quantification made closest to the time of the interview, using the NASBA technique; the data was obtained from the patients' records.

Flow cytometry $\mathrm{CD}_{4}$ counts were copied from the patients' records. The most recent $\mathrm{CD}_{4}$ counts were used as an indication of the current state of the immune system. Figures lower than 200 have been considered indicators of serious immunological debilitation [42]. The lowest $\mathrm{CD}_{4}$ figure of each patient was used as part of the criterion for classifying the clinical stage of infection. Clinical stage of HIV infection was estimated according to CDC (Centers for Disease Control and Prevention) criteria [43]; patients were classified as: stage A, asymptomatic; stage B, having symptoms of HIV infection but without criteria for diagnosis of the disease; and stage $\mathrm{C}$, having diseases that define AIDS (Kaposi's sarcoma, for example), with a total of 10 or more points according to the Caracas criterion [42]. The $\mathrm{CD}_{4}$ counts and viral load counts were extracted from the patient's records for this purpose.

\section{Aspects of treatment and other clinical information.} The following variables were obtained from the patients' records: time of diagnosis of HIV/AIDS carrier, date when antiretroviral treatment was initiated, current antiretroviral regimen, and other antiretroviral regimens previously used by patients (including names of medications, number of daily doses, number of pills per treatment session, compulsory fasting or medication taken with a meal), adverse effects and use of other medications.

Perception of negative effects and negative physiologicalstates. These perceptions were measured by means of a scale developed for this study. This scale includes five items: "I have been sad and feeling low," "I have been nervous and irritable," "I have been feeling bad," "I have been having problems with my day-today activities," "I feel debilitated and sick." A Likert scale was added to each item, where the patient expressed the degree of agreement with the situation described by the item. Items were classified as (o):"strongly disagree", (1) “disagree”, (2) “don't know”, (3)"agree”, (4) “agree strongly." A score of perception of negative effects and negative physiological states was generated after the first main component of the exploratory analysis of the main components. This score was designed in a standardized z-scale and measured, in standard deviations, how much the individual's perception was above or below the sample mean. This scale showed acceptable levels of internal consistency, as indicated by a Cronbach's alpha value of 0.75 .

Adherence to antiretroviral regimen during the study period. The use of antiretrovirals was investigated at first and second interviews (interval of approximately one month between them) by means of a self-report, using a questionnaire that investigated the medications in use, schedule of administration and doses during the previous 48 hours.

Adherence to the regimen during this period was estimated as follows: initially, adherence to each separate medication was calculated by dividing the number of pills that the patient declared he took by the number of pills prescribed (first and second interview). After that, adherence to the regimen during this period was estimated by an arithmetic mean of adherence to medications. A patient was considered adherent if he or she took $\geq 95 \%$ of the prescribed dose during the four days of evaluation. 
Statistical analyses. Percentages of adherent patients and crude and adjusted odds ratios of adherence were estimated for each variable of the treatment regimen. Gender, clinical stage, time since diagnosis, and duration of treatment were not considered potential confounding factors because they did not show evidence of any association with adherence (they all presented $p>0.30$, both in the crude model and in the model adjusted for age, family income, schooling, perception of negative effects and negative physiological states). Age, family income, schooling, perception of negative effects and negative physiological states were included as potential confounding factors of the effect of treatment regimen on adherence (they all gave a significant correlation with adherence, $\mathrm{p}<0.05)$. It was determined that all variables of treatment regimen that had any significant or nearly significant effect on adherence $(p<0.30)$, either in the crude model or in a model adjusted for age, family income, schooling, perception of negative effects and negative physiological states, would also be included as potential confounding factors for the other variables of this regimen. The only exception was the number of doses taken in a fasting state, which was not included in the other models because it was too closely related to the number of daily doses.

\section{Results}

Two hundred and forty four patients eligible for the study were registered at SAE. Among those, $10 \mathrm{had}$ died, 27 were not found and 194 participated in the study. Most $(60.8 \%)$ of the patients who participated were men. Their ages ranged from 17 to 67 years, with a mean of 34 . Schooling varied from 0 to 15 years, with a mean of 5 years; family income ranged from 0 to 46 minimum wages, and it was less than 1.5 minimum wages in $50 \%$ of the patients. The time since diagnosis ranged from 0 to 9 years, with a mean of 2 years. A third of all patients had less than six months of treatment. Most of the patients $(60.3 \%)$ had stage $\mathrm{C}$ disease and $28.4 \%$ had stage A. The current immune status of $73.7 \%$ of patients was good, which was evidenced by $\mathrm{aCD}_{4}$ count of $>200$ cells $/ \mathrm{ml}$. Viral load count was $<400$ copies $/ \mathrm{ml}$ in $64.7 \%$; $49 \%$ of the patients had adhered to treatment.
More than half of the patients used four or more pills per session, three or four drugs in each regimen, three or more daily doses, and one or more doses administered in a fasting state. Most patients never changed their antiretroviral regimen, never used other medications and did not report adverse effects to antiretroviral treatment (Table 1). The vast majority of antiretroviral regimens with more than two daily doses $(88.9 \%$; $n=111)$ required at least one dose administered in a fasting state.

Patients with a prescription of three to four daily doses were $53 \%$ less likely to adhere (odds ratio of adherence to treatment $(\mathrm{OR})=0.47,95 \%$ confidence interval (CI) 0.22-1.01) than patients who had been prescribed a two-dose regimen; for those who had been prescribed 5 to 6 doses, the likelihood of adherence was $76 \%$ lower $(\mathrm{OR}=0.24,95 \%$ CI $0.09-0.62)$, after adjustments for age, family income, schooling, use of other medications and perception of negative effects, negative physiological states and adverse effects (Table 1).

Two or more daily doses taken in a fasting state reduced the likelihood of adherence by $73 \%$, when compared with absence of compulsory fasting (OR=0.27, 95\% CI 0.11-0.68), adjusted for age, family income, schooling, perception of negative effects, negative physiological states, adverse effects and use of other medications (Table 1).

The perception of adverse effects reduced the likelihood of adherence by $61 \%(\mathrm{OR}=0.39,95 \% \mathrm{CI}$ 0.19-0.77) as compared with the absence of these effects, after adjustments for age, family income, schooling, perception of negative effects, negative physiological states, adverse effects and use of other medications (Table 1). The most frequent adverse effects were gastrointestinal symptoms $(n=47)$, mood swings, thinking and behavioral changes $(n=15)$, neurological symptoms $(\mathrm{n}=13)$ and headaches and diffuse pain $(\mathrm{n}=10)$.

The use of other combined medications did not affect the adherence in the crude model $(\mathrm{p}=0.363)$. However, after adjustments for age, family income, schooling, perception of negative effects, and negative physiological states, the likelihood of adherence was reduced by $42 \%$ compared with those who did not have other medications prescribed $(\mathrm{OR}=0.58 ; 95 \%$ 
Table 1. Number of patients, percentages of individuals adhering to treatment, and Odds Ratio of adherence to antiretrovirals

\begin{tabular}{ccccc}
\hline Variables & Patient & Adherence & OR (CI 95\%) & OR (CI 95\%) \\
& Adjusted
\end{tabular}

Maximum number of tablets

perdose

$\begin{array}{cccc}1-3 & 69 & 49.3 & 1 \\ 4 & 85 & 49.4 & 1.01(0.53-1.97) \\ \geq 5 & 40 & 46.5 & 0.93(0.43-2.03) \\ & & & \mathrm{p}=0.987\end{array}$

Number of drugs per Regimen

2
3
4

Daily Doses (1)

$$
2
$$

3 a 4

5 a 6

Administered with a meal

No

Yes

Administered in a fasting state (1)

None

1

$\geq 2$

Change of Regimen

No

26

152

16

34.6

51.3

50.0

69

79

46

177

17

48.6

52.9

82

68

44

59.8

47.1

31.8

119

84

48.7

49.3

110

84

57.8

45.2

No

Adverse Effects (3)

Not reported

Reported
$118 \quad 57.6$

$\begin{array}{ll}76 & 35.3\end{array}$

35.3
Other medications (2)

$$
\begin{array}{cc}
1 \\
2.01 \quad(0.83-4.74) \\
1.89 \quad(0.53-6.72) \\
\mathrm{p}=0.283
\end{array}
$$

\section{3}

1

$0.51(0.26-0.98)$

$0.47(0.22-1.00)$

$0.32(0.15-0.70)$

$\mathrm{p}=0.011$

$0.24(0.09-0.62)$

$\mathrm{p}=0.007$
$1.20(0.44-3.22)$

$$
\mathrm{p}=0.731
$$

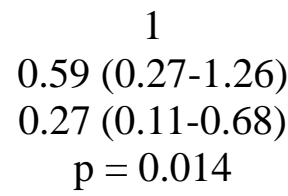

$0.60(0.31-1.14)$

$0.31(0.15-0.69)$

$\mathrm{p}=0.010$

$\mathrm{p}=0.014$

(1) Adjusted for age, family income, schooling, perception of negative effects, negative physiological states, adverse effects, and use of other medications; (2) Adjusted for age, family income, schooling, perception of negative effects, negative physiological states, daily doses, and use of other medications; (3) Adjusted for age, family income, schooling, perception of negative effects, negative physiological states, daily doses, and use of other medications. 
CI 0.30-1.10; p=0.092) (data not shown), and therefore it was included as a confounding factor of other variables of the therapeutic regimen (Table 1).

Percentages of adherence were similar among various categories of variables, including maximum number of pills administered per session, the number of drugs in each treatment regimen, medication administered with a meal, and changes in the treatment regimen. There was no evidence of a significant effect of these variables on adherence (crude and adjusted odds ratio; $p>0.283$ ) (Table 1 ).

\section{Discussion}

Currently there is no gold standard for measuring adherence $[13,18,41]$; some methods of doing so include interviews with patients, manual counting of pills, counting of opened bottles and serum or urinary dosing of drug metabolites [14,39]. Interviews [15,41] and counting of pills $[13,14,22,29]$ tend to overestimate adherence, but their main advantages are low cost and readiness to obtain the desired information $[13,14,36]$. Dosing of metabolites can be affected by the medication's half-life $[13,44]$; therefore, it does not offer accurate information about the last dose taken by the patient [39]. Electronic counting is expensive and tends to overestimate adherence because patients may open the bottles and not take the tablets [13]. In our trial, prevalence of adherence was evaluated by means of a self-report from the patient. Nowadays, this is the most widely used method $[7,8,12,15,16,21,40,41,45,46]$, and it has been useful to identify patients at risk for nonadherence and to forecast the results of short-term treatment as assessed by viral load counts $[7,8,25,41]$.

Adherence during this period was estimated by the information collected in the two interviews regarding the mean use of antiretrovirals; each interview involved medication taken during the previous 48 hours. Adherence was defined as the use of $\geq 95 \%$ of the prescribed dose, a level that was recommended by the Brazilian Ministry of Health [1], as it is associated with therapeutic effectiveness. The vast majority of older studies used a cut-off point of $80 \%[11,13,16,30]$. Nowadays, some studies use even more stringent cutoff points of as high as $100 \%[21,25]$.

This study was carried out in a reference service of the Unified National Health System (SUS, Sistema Único de Saúde) for HIV/AIDS in the city of Pelotas, where $79.5 \%$ of the patients registered in that service participated in our study. Adherence to drug regimens was low (49\%) compared with another study that found $69 \%$ adherence and also used $95 \%$ of the prescribed medication as a cut-off for defining adherence [7]. Low adherence might be explained in part because of the low level of schooling of the patients from SAE in the city of Pelotas.

Our research about the effects of the characteristics of the treatment regimen on adherence had a crosssectional design; we were unable to establish causal relations between these variables and treatment adherence. Nevertheless, in agreement with other studies, we suggest that regimens with three or more daily medication doses, with two or more doses taken in a fasting state, or those which produce adverse effects noticed by the patient (such as gastrointestinal problems, mood swings, thinking and behavioral changes, neurological effects and diffuse pain) tend to reduce treatment adherence. In our study, the amount of medication taken in a fasting state was very much related to the number of daily doses, and it was impossible to measure the effect of one separately from the other. Other studies and reviews also found an association between non-adherence and the number of daily doses [16,18,19,28], compulsory fasting [19,24,32-34], and adverse effects of treatment [4,18,19,34-38].

One study indicated that patients who took four drugs had a less rigorous adherence than those who took only one or two drugs [25]. In our study, regimens using three and four drugs had a likelihood of adherence approximately two times lower than that seen in twodrug regimens, but this effect was not significant, probably due to the low power of this study to detect effects of such magnitude. Studies with larger sample sizes are necessary to examine the association that between adherence and the number of drugs used. 
Parameters such as the maximum number of pills per medication session, changes in the antiretroviral regimen and compulsory administration with food did not reveal any relationship with adherence ( $p$ values $>0.30$ and effect $\leq 30 \%$ ). Other studies also found no association between treatment adherence and the number of pills per medication session [4,11-13,15,31,39] or with changes in the antiretroviral regimen [25].

Based on our study, we suggest a need for strategies to increase treatment adherence. Efforts must be made towards simplifying the therapeutic regimen, to reduce the number of daily doses and substituting with regimens that are accompanied by fewer adverse effects, wherever possible [14,18,47]. Emphasis on instruction of patients to take the medication is fundamental, as well as patient's participation in some treatment decisions [12,18,22,29]. It has been recommended that the physician discuss with the patient potential adverse effects and means to minimize them [14,19,21,22,29,47]. It is also important to stimulate the patient to implement the therapeutic regimen in his or her daily routine, pointing out, at each consultation, the importance of adherence. Adherence groups, as well as the participation of friends and family members have also been recommended as potentially beneficial for adherence [14,29,47]. It is crucial to explain to those patients whose medications need to be taken in a fasting state, that the absorption of the drug is reduced when there is food in the digestive tract $[22,29,33]$.

\section{Acknowledgements}

This paper originated from a Master's thesis by Vera Lúcia da Silveira. The authors thank the Fundação de Amparo à Pesquisa do Estado do Rio Grande do Sul (FAPERGS - "Research Support Foundation of the State of Rio Grande do Sul" - Act number 990161.2) for its support of José Carlos de Carvalho Leite, the researcher responsible for the investigational research trial Motivação para o tratamento como preditor da adesão aos antiretrovirais no sul do Brasil ("Motivation for treatment as a predictor of adherence to antiretrovirals southern Brazil"'), which gave rise to this study, and the Conselho Nacional de Pesquisa (CNPq) (National Council of Scientific and Technological Development) for financial assistance.

\section{References}

1. Ministério da Saúde do Brasil. Recomendações para Terapia Anti-retroviral em adultos e adolescentes infectados pelo HIV Brasil. 2001.

2. Rashid M., Schechter M. Manual de HIV/AIDS 2001;6:4-5.

3. Bartlett J.G.., Gallant J.E. Medical Management of HIV infection. 2000:39.

4. Monforte A.A., Testa L., Adorni F., et al. Clinical outcome and predictive factors of failure of highly active antiretroviral therapy in antiretroviral-experienced patients in advanced stages of HIV-1 infection. AIDS 1998; $12: 1631-7$.

5. Paterson D.L., Swindells S., Mohr J., et al. Adherence to protease inhibitor therapy and outcomes in patients with HIV infection. Ann Intern Med 2000;133:21-30.

6. Vanhove G.F., Schapiro J.M., Winters M.A., et al. Patient compliance and drug failure in protease inhibitor monotherapy. JAMA 1996;276:1955-6.

7. Haubrich R.H., Little S.J., Susan J., et al. The value of patient-reported adherence to antiretroviral therapy in predicting virologic and immunologic response. AIDS 1999;13:1099-107.

8. Bangsberg D.R., Hetcht F.M., Charlebois E.D., et al. Adherence to protease inhibitor, HIV-1 viral load, and development of drug resistance in an indigent population. AIDS 2000; 14:357-66.

9. Lucas G.M., Chaison R.E., Moore D. Highly active antiretroviral therapy and adverse drug reactions. Ann Int Med 1999;131:81-7.

10. Tchetgen E., Kaplan E.H., Friedland G.H. Public health consequences of screening patients for adherence to highly active antiretroviral therapy. JAIDS 2001;26:118-29.

11. Singh N., Squier C., Sivek C., et al. Determinants of compliance with an antiretroviral therapy in patients with human immunodeficiency virus: prospective assessment with implications for enhancing compliance. AIDS CARE 1996;8:261-9.

12. Singh N., Berman S., Stephen M., et al. Adherence of human immunodeficiency virus- infected patient to antiretroviral therapy. Clin Infect Dis 1999;29:824-30.

13. Nemes M.I.B., Souza M..M., Souza A., et al. Prevalência da aderência e fatores associados. In: Ministério da Saúde do Brasil, Coordenação Nacional de DST-AIDS (Eds). Aderência ao tratamento por anti-retrovirais em Serviços Públicos no Estado de SP 2000:65-101.

14. Besh C.L. Compliance in Clinical Trials. AIDS 1995;9:1-10.

15. Haynes R.B., Mckibbon K.A, Kanani R. Systematic review of randomized trials of interventions to assist patients to follow prescriptions for medications. The Lancet 1996;348:383-6. 
16. Eldred L.J., Wu A.W., Chaisson R.E., et al. Adherence to antiretroviral and Pneumocystis carini prophylaxis in HIV disease. Journal of Acquired Syndromes and Human Retrovirology 1997; 18:117-25.

17. Mehta S., Moore R.D., Grahan N..M. Potential factors affecting adherence with therapy. AIDS 1997;11:1665-70.

18. Ickovics J.R., Meisler A.V. Adherence in Aids clinical trials: a framework for clinical research and clinical care. J Clin Epidemiol 1997;50:385-91.

19. Gallant J.E., Block D.S. Adherence to antiretroviral regimens in HIV-infected patients: results of a survey among physicians and patients. Journals of the International Association of Physicians in Aids Care 1998:32-5.

20. Altice F., Friedland G.H. The era of adherence to HIV therapy. Ann Intern Med 1998; 129:503-5.

21. Kalichman S.C., Ramachandran B.B., Catz S. Adherence to combination antiretroviral therapies in HIV patients of low health literacy. J Gen Intern Med 1999; 14:267-73.

22. Sherr L. Understanding adherence. J HIV Ther 2000;5:30-5.

23. Morse E.V., Simon P.M, Coburn M., et al. Determinants of subject compliance within in experimental anti-HIV drug protocol. Soc Sci Med 1991;32:1161-7.

24. Mallow R.M., McPherson S., Klimas N., et al. Adherence to complex combination antiretroviral therapies by HIVpositive drugs abusers. Psychiatr Serv 1998;49:1021-2.

25. Kleeberger C.A, Phair J.P., Strathdee S.A., et al. Determinants of heteregenous adherence to antiretroviral therapies to $\mathrm{HIV}$-antiretroviral in the multicenter Aids cohort study. JAIDS 2001;26:118-9.

26. Sackett D.L., Snow J.C. The magnitude of compliance and noncompliance. In Haynes R.B., Taylor D.W., Sackett D.L (Eds). Compliance in health care 1979;11-22.

27. Haynes R.B., Wang E., Gomes M.M. A critical review of interventions to improve compliance with prescribed medications. Patient education and counseling 1987; 10:155-66.

28. Kastrissios H., Suárez J.R., Katzenstein D., et al. Characterizing patterns of drug-takins behavior with a multiple drug regimen in AIDS clinical trial. AIDS 1998; 12:2295-303.

29. Willians A., Friedland G. Adherence, compliance and HAART. AIDS Clin Care 1997; 7:51-8.

30. Samet J.H., Libman H., Steger K.A., et al. Compliance with Zidovudine therapy in patients infected with human immunodeficiency virus, type 1: a cross-sectional study in a municipal hospital clinic. Am J Med 1992;92:495-502.

31. Mostashari F., Riley E., Slwyn P.A., Altice F.L. Acceptance and adherence with antiretroviral therapy among HIVinfected women in a correctional facility. Journal of Acquired Immune Deficiency Syndromes and Human Retrovirology 1998; 18:341-8.
32. Kemper C.A., Linnet A., Kane C., et al. Travels with HIV: the compliance and health of HIV-infected adults who travel. Int J STD AIDS 1997;8:44-9.

33. Garey K.W., Rodvold K. Antiretroviral agents and food interactions. Infect Med 1998; 15:863-9

34. Max B., Sherer R. Managements of the adverse effects of antiretroviral therapy and medication adherence. Clin Infect Dis 2000;30:96-116.

35. Muma R.D., Ross M.W., Parcel G..S., et al. Zidovudine adherence among individuals with HIV infection. AIDS CARE 1995; 7:439-47.

36. Mehta S., Moore R., Grahan N.M.H.. Potential factors affecting adherence with HIV therapy. AIDS 1997; 11:1665:70.

37. Koop C.E. Assessing and enhancing compliance with antiretroviral therapy. The nurse practitioner 1997;22:311-9.

38. Belzer M., Slonimsky G., Tucker D. Antiretroviral adherence issues among HIV + youth. J Adolesc Health 1998;22:160.

39. Broers B., Morabia A., Hirshel B. A cohort study of drug users' compliance with Zidovudine treatment . Arch Intern Med 1994;154:1121-7.

40. Gordillo V., Amo J., Soriano V., Lahoz J.G. Sociodemographic and psychological variables influencing adherence to antiretroviral therapy. AIDS 1999; 13:1763-9.

41. Chesney M.A., Ickovics J.R., Chambers D.B., et al. Selfreported adherence to antiretroviral medication among participants in HIV clinical trial: the AACTG adherence instruments. AIDS CARE 2000; 12 :255-6.

42. Centers for Disease Control and Prevention. Report of the $\mathrm{NIH}$ panel to define principles of therapy of HIV infection and Guidelines for the use of antiretroviral agents in HIVinfected adults and adolescents TM US. Department of Health and Human Services. Atlanta 2000.

43. Center for Disease Control and Prevention. Revised classification system for HIV infection and expanded surveillance case definition for AIDS among adolescent and adults. MMWR 1993;41:1-19.

44. Kasstrissios H., Suárez J.R., Hammer S. The extent of nonadherence in a large AIDS clinical trial using plasma dideoxynucleoside concentrations as a marker. AIDS 1999; 12:2305-11.

45. Steiner J.F., Colo D. Provider assessments of compliance with Zidovudine. Arch Intern Med 1995;155:335-6.

46. Murri R., Ammassari A. Gallicano K., et al. Patient-reported non adherence to HAART is related to protease levels. JAIDS 2000;24:123-8

47. Leake H., Horne R. Optimising adherence to combination therapy. J HIV Ther 1998;3:67-71. 